\title{
Modified Yi Guan Jian, a Chinese herbal formula, induces anoikis in Bel-7402 human hepatocarcinoma cells in vitro
}

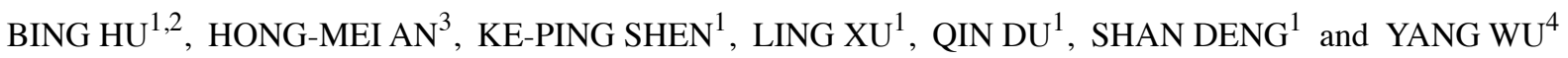 \\ ${ }^{1}$ Department of Oncology and Institute of Traditional Chinese Medicine in Oncology, Longhua Hospital, \\ ${ }^{2}$ E-institute of Internal Medicine of Traditional Chinese Medicine, Shanghai Municipal Education \\ Commission, and ${ }^{3}$ Department of Science and Technology, Longhua Hospital, Shanghai University of \\ Traditional Chinese Medicine, Shanghai; ${ }^{4}$ State Key Laboratory of Biotherapy and Cancer Center, West \\ China Hospital, West China Medical School, Sichuan University, Chengdu, Sichuan, P.R. China
}

Received June 14, 2011; Accepted July 21, 2011

DOI: 10.3892/or.2011.1414

\begin{abstract}
Liver cancer is the fifth most common malignancy worldwide. Liver YIN deficiency is a common clinical syndrome of traditional Chinese medicine in liver cancer. Yi Guan Jian is an ancient classic liver YIN tonifying herbal formula used for the treatment of liver disease with liver YIN deficiency, which is also currently used for liver cancer treatment. However, as an ancient formula, Yi Guan Jian (YGJ) is not entirely suitable for liver cancer treatment. In the present study, we optimized the prescription of YGJ according to the current principles of Chinese herbal medication, and evaluated the anticancer effects of modified Yi Guan Jian (MYGJ) in Bel-7402 human hepatocarcinoma cells. The results show that MYGJ inhibited the growth of Bel-7402 cells in adherent or in suspension cultures, and was more effective in Bel-7402 cells in suspension. MYGJ also inhibited anchorageindependent growth of Bel-7402 cells in soft agar. MYGJ induced anoikis in Bel-7402 cells accompanied by caspase-3, -8 and -9 activation, which was blocked by the pan-caspase inhibitor, Z-VAD-FMK. Furthermore, MYGJ inhibited the expression and phosphorylation of p38 MAPK in Bel-7402 cells. These findings suggest that MYGJ is sufficient to induce caspase-mediated anoikis in Bel-7402 cells in vitro, and may be associated with down-regulation of p38 MAPK. The present study also provides insight into the application of ancient Chinese herbal formulas.
\end{abstract}

\section{Introduction}

Liver cancer is the fifth most common malignancy worldwide and the second most common cause of death from

Correspondence to: $\mathrm{Dr}$ Bing Hu, Department of Oncology, Longhua Hospital, Shanghai University of Traditional Chinese Medicine, 725 South Wanping Road, Shanghai 200032, P.R. China E-mail: beearhu@hotmail.com

Key words: hepatocarcinoma, Chinese herbal formula, anoikis, caspases, MAPK cancer (1). The incidence of liver cancer is increasing due to the dissemination of hepatitis $\mathrm{B}$ and $\mathrm{C}$ virus infections, and the highest incidence has been recorded in East and SouthEast Asia and in Middle and Western Africa (1,2). Currently, the treatment of hepatic carcinoma is mainly dependent on early surgical resection, liver transplantation, chemoembolization, and targeted therapy $(2,3)$. However, most patients present with middle or advanced stages at diagnosis and not amenable to loco-regional therapy, due to the latent onset and rapid progression $(3,4)$. Treatment of middle-advanced stage liver cancer is difficult because of relatively few therapeutic responses and survival benefit. In addition, the overall efficacy of recently approved targeted therapy (sorafenib) remains moderate (5). It is important to develop novel therapeutic methods to treat liver cancer.

In China, traditional Chinese medicine has been widely used in all aspects of integrative therapy in liver cancer, including surgery, liver transplantation, chemoembolization, targeted therapy, and even treatment alone for middle-advanced stage liver cancer (6-9). It has been reported that traditional Chinese medicine may help to delay tumor progression, improve quality of life, and prolong survival in liver cancer patients (6-9). The deficiency of liver-YIN is a common clinical syndrome of traditional Chinese medicine in liver cancer, especially in middle-advanced stage liver cancer $(10,11)$. Yi Guan Jian (YGJ), a classic liver-YIN tonifying herbal formula, was established by Wei ZX in the Qing Dynasty (AD 1722-1772), first recorded in Medical talks in Liu Zhou, and initially used for treating liver disease with liver-YIN deficiency. Currently, YGJ is also frequently used to treat liver cancer with liver-YIN deficiency $(12,13)$.

The original prescription of YGJ was composed of coastal glehnia root, ophiopogonis tuber, angelica, dried rhizome of rehmannia, wolfberry fruit and fructus toosendan. It has been reported that some of these herbs or related components possess a certain degree of anti-cancer potential, such as coastal glehnia root, ophiopogonis tuber, angelica, wolfberry fruit, fructus toosendan, or components in these herbs (14-17). However, as a tonifying herbal formula, the herb dosages in original prescription of YGJ are not entirely applicable 

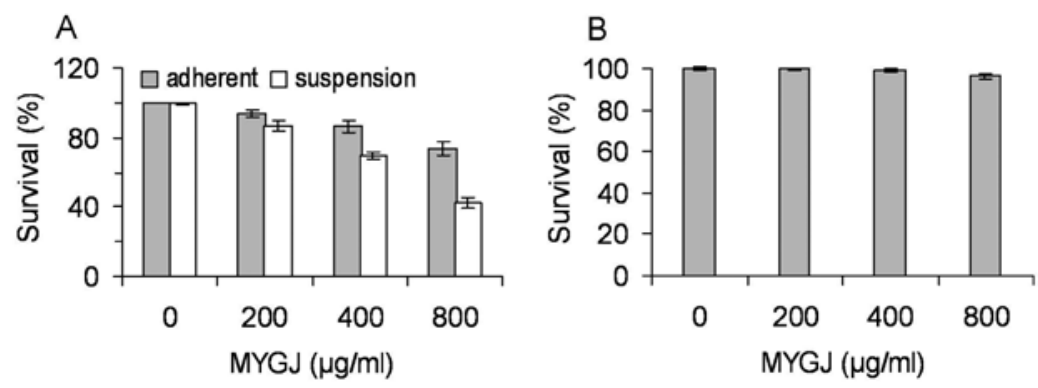

Figure 1. Effects of MYGJ on the proliferation of Bel-7402 and HL-7702 cells. Human heptocarcinoma Bel-7402 cells (A) and human normal hepatocyte HL-7702 (B) were treated with different concentrations of MYGJ for $24 \mathrm{~h}$, cell viability was evaluated by Calcein AM fluorescence assay. Data shown are representative of three independent experiments.

to middle-advanced stage liver cancer, and the anti-cancer effects of YGJ are insufficient. In this study, we optimized the prescription of YGJ according to the current principles of Chinese herbal medication, and evaluated the anti-cancer potential of modified Yi Guan Jian (MYGJ) in human hepatocarcinoma Bel-7402 cells. Our results show that MYGJ may inhibit suspension growth, activate caspases, and induce anoikis in Bel-7402 cells, and may relate to MAPK expression and phosphorylation.

\section{Materials and methods}

Chemicals and reagents. CytoSelect ${ }^{\mathrm{TM}}$ 24-well anoikis assay was provided by Cell Biolabs (San Diego, CA). Calcein AM and poly-HEMA was from Sigma-Aldrich (St. Louis, MO). Colorimetric CaspACE ${ }^{\mathrm{TM}}$ Assay System was the product of Promega (Madison, WI). Z-VAD-FMK, caspase-8 and caspase-9 Colorimetric Assay kits were from R\&D Systems (Minneapolis, MN). Antibodies against p38 MAPK, phosphop38 MAPK, and GAPDH were obtained from Cell Signaling Technology (Danvers, MA).

Herbal preparation. MYGJ was prepared as a lyophilized-dry powder of hot water extracts as previously described (18-20). Authentic herb materials were provided by Longhua Hospital herb store. Herbs used are coastal glehnia root (15 g), ophiopogonis tuber $(15 \mathrm{~g})$, angelica $(9 \mathrm{~g})$, dried rhizome of rehmannia $(9 \mathrm{~g})$, wolfberry fruit $(15 \mathrm{~g})$, fructus toosendan $(6 \mathrm{~g})$, and polygonum cuspidatum $(15 \mathrm{~g})$. All these herbs were soaked for $1 \mathrm{~h}$, and decocted twice with 8-fold volume of distilled water for $2 \mathrm{~h}$. The decoction was filtered and centrifuged twice at $12000 \mathrm{rpm}$ for $30 \mathrm{~min}$ to remove insoluble ingredients. The supernatants were mixed with an equal volume of ethanol and kept at $4^{\circ} \mathrm{C}$ overnight, and centrifuged at $12000 \mathrm{rpm}$ for $30 \mathrm{~min}$ to remove insoluble ingredients. The resultant supernatants were lyophilized, weighed, dissolved in RPMI-1640 medium and adjusted to a concentration of $400 \mathrm{mg} / \mathrm{ml}$, and was sequentially passed through 0.45 and $0.22 \mu \mathrm{m}$ filters for sterilization.

Cell culture. Human hepatocarcinoma cell line Bel-7402 and human hepatocyte cell HL-7702 was obtained from Cell Bank of Type Culture Collection of Chinese Academy of Sciences. Bel-7402 and HL-7702 cells were grown in RPMI-1640 medium with $10 \%$ FBS and $1 \%$ penicillin-streptomycin, and maintained at $37^{\circ} \mathrm{C}$ in a humidified incubator with a $5 \% \mathrm{CO}_{2}$ atmosphere.

Cell proliferation assay. Cells in logarithmic growth phase were seeded into poly-HEMA coated $(10 \mathrm{mg} / \mathrm{ml})$ or uncoated 96-well plate $\left(5 \times 10^{3}\right.$ cells/well). After $24 \mathrm{~h}$ cells were exposed to various doses of MYGJ for $24 \mathrm{~h}$, and cell viability was evaluated by using the Calcein AM fluorescence assay according to the manufacturer's instructions. The cell survival rate was calculated as follows: cell survival rate $(\%)=$ experimental OD value/control OD value x $100 \%$.

Anchorage-independent growth assay. For the soft agar colony formation assays, $2 \times 10^{4} \log$-phase Bel-7402 cells were seeded and grown on a plate containing $1 \%$ base agar and $0.6 \%$ top agar, and exposed to different concentrations of MYGJ twice a week for 2 weeks and incubated at $37^{\circ} \mathrm{C}$ in a humidified incubator with a $5 \% \mathrm{CO}_{2}$ atmosphere. Colonies were stained with crystal violet and counted under a dissecting microscope.

Anoikis assay. Anoikis was detected by CytoSelect ${ }^{\mathrm{TM}}$ 24-well anoikis assay according to the manufacturer's instructions. Briefly, log-phase Bel-7402 cells ( $3 \times 10^{4}$ cells/well) were inoculated in poly-HEMA coated 24-well plate. On the second day, the cells were exposed to a different dose of MYGJ or equal volume of RPMI-1640. After 24-h incubation with the MYGJ, ethidium homodimer (EthD-1) was added into each well and incubated at $37^{\circ} \mathrm{C}$ for $1 \mathrm{~h}$. The presence of red EthD-1 fluorescence was monitored under a fluorescence microscope, and quantitated with a fluorescence microplate reader.

Flow cytometric assays. Cells were treated as indicated, collected, and stained with Annexin V-FITC and PI as recommended by the manufacturer (BD Biosciences). Apoptotic cells were detected in a FACScalibur flow cytometer (BectonDickinson).

Caspase activation assay. After treatment for the indicated time with different concentration of MYGJ, caspase-3, -8, -9 activities were measured by the cleavage of the specific chromogenic substrate according to the manufacturer's instructions. For caspase inhibition, cells pretreated with Z-VAD-FMK $(50 \mu \mathrm{mol} / 1,2 \mathrm{~h})$ were incubated with various concentration of MYGJ $(20 \mu \mathrm{g} / \mathrm{ml})$ for another $24 \mathrm{~h}$. 


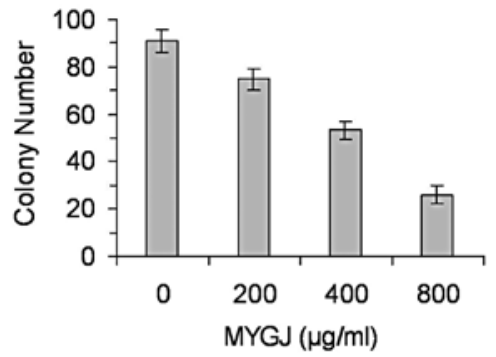

Figure 2. Effects of MYGJ on anchorage-independent growth of Bel-7402 cells. Bel-7402 cells were treated with MYGJ $(200-800 \mu \mathrm{g} / \mathrm{ml})$ twice a week for 2 weeks in the soft-agar colony formation assay. The colonies were stained and counted. Data shown are representative of three independent experiments (each conducted in triplicate).

Western blot analysis. Western blots were performed as previously described (19). Briefly, collected cells were lysed and subjected to $12 \%$ SDS-PAGE gel, and transferred onto a nitrocellulose membrane (Amersham). The transferred membrane were blocked with $5 \%$ non-fat milk, washed, and probed with the indicated antibodies. Blots were then washed and incubated with IRDye 700- and IRDye 800-conjugated secondary antibodies (Rockland Immunochemicals), and visualized in Odyssey Infrared Imaging System (LI-COR Biosciences).

Statistical analyses. Results are expressed as means \pm standard deviation of at least two independent experiments, each conducted in triplicate. Differences between control and MYGJ treatment were analysed by one-way ANOVA. Differences were considered significant at $\mathrm{P}<0.05$.

\section{Results}

MYGJ inhibited proliferation of Bel-7402 cells. The effects of MYGJ on cell growth of human heptocarcinoma Bel-7402 cells adherent or in suspension were detected by using poly-HEMA uncoated or coated plates. The results shown that MYGJ, at a concentration of $200-800 \mu \mathrm{g} / \mathrm{ml}$, significantly inhibited proliferation of Bel-7402 cells in adherent and suspension in a dose-dependent manner (Fig. 1A) $(\mathrm{P}<0.05)$. To our surprise, MYGJ was more sensitive to suspension-cultured Bel-7402 cells. Meanwhile, human normal hepatocyte HL-7702 cells were used as a control. MYGJ, at a final concentration of $200-800 \mu \mathrm{g} / \mathrm{ml}$, showed no significant effects on the growth of normal hepatocytes HL-7702 cells (Fig. 1B).

MYGJ inhibited colony formation of Bel-7402 cells in soft agar. Based on the effects of MYGJ on proliferation of Bel-7402 cells in suspension, effects of MYGJ on long-term anchorage-independent growth of Bel-7402 cells were further observed by using the characteristics of cell growth in soft agar without adherence. Soft agar colony formation assay shown that MYGJ, at a final concentration of $200-800 \mu \mathrm{g} / \mathrm{ml}$, significantly inhibited colony formation of Bel-7402 cells in soft agar (Fig. 2) $(\mathrm{P}<0.05)$, which suggested that MYGJ might inhibit anchorage-independent growth of Bel-7402 cells.

MYGJ induced anoikis in Bel-7402 cells. EthD-1, a red fluorescent dye, was used to detect cell death in suspension culture. As shown in Fig. 3A and B, after MYGJ treatment, EthD-1 was absorbed by Bel-7402 cells yielding a red-fluorescent nuclear staining, and nuclear fragmentation was observed in some cells. In addition, the photodensity of the red fluorescence between MYGJ, in different dosage and control groups, showed a significant difference $(\mathrm{P}<0.01)$. Flow cytometry analysis was used to further discriminate necrosis and apoptosis. As shown in Fig. 3C, MYGJ significantly induced apoptosis in Bel-7402 cells in suspension culture $(\mathrm{P}<0.01)$. These results suggested MYGJ may induce anoikis in Bel-7402 cells.

MYGJ activated caspases in Bel-7402 cells. Activation of caspases has been recognized as hallmarks of apoptosis. Anoikis, a type of apoptosis in suspension, is also executed
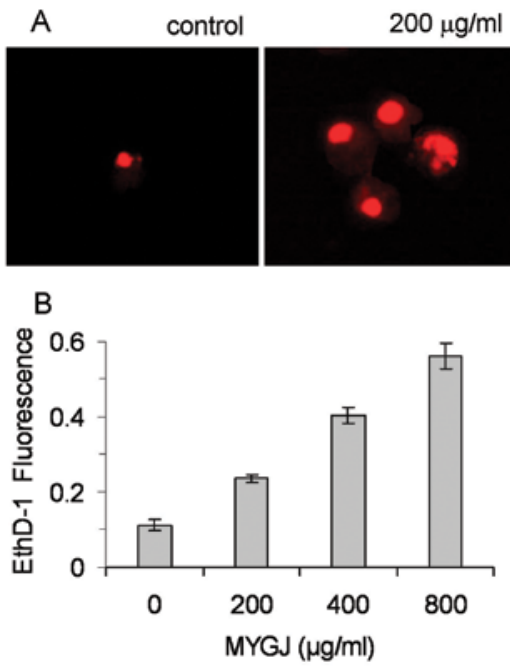

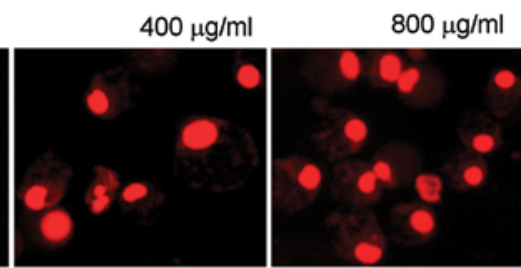

C

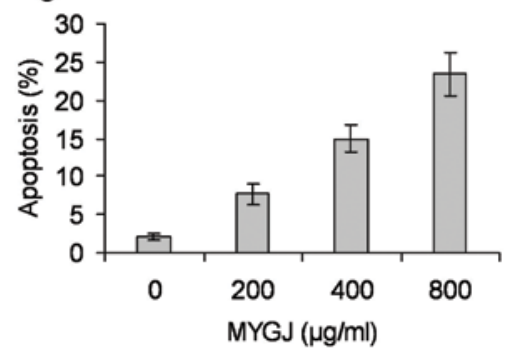

Figure 3. MYGJ induces anoikis in Bel-7402 cells. (A) Bel-7402 cells were treated with different dose of MYGJ for 24 h in poly-HEMA coated plates, stained with EthD-1, and observed under fluorescence microscope (x100). (B) The fluorescence of EthD-1 absorbed by Bel-7402 cells was detected at 525 nm with fluorescence microplate reader, and expressed as mean \pm SD. (C) MYGJ treated Bel-7402 cells were stained with Annexin V-FITC/PI and detected in FACScalibur flow cytometer. Data illustrated are from three separate experiments. 

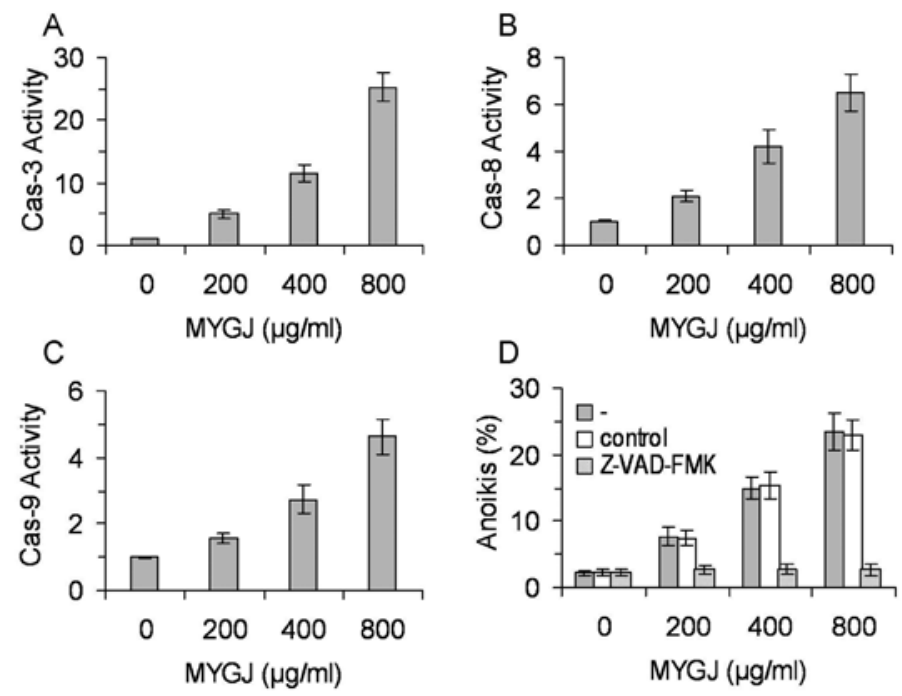

Figure 4. MYGJ activates caspases in Bel-7402 cells. After $24 \mathrm{~h}$ of MYGJ (200-800 $\mu \mathrm{g} / \mathrm{ml})$ treatment, caspase-3 (A), caspase-8 (B), caspase-9 (C) activity in suspension-cultured Bel-7402 cells were detected as described in Materials and methods. Caspase activities are expressed as fold activation over control. (D) Suspension-cultured Bel-7402 cells were pretreated with Z-VAD-FMK $(50 \mu \mathrm{mol} / \mathrm{l})$ for $2 \mathrm{~h}$ before treatment with MYGJ for $24 \mathrm{~h}$, stained with Annexin V-FITC/PI and analyzed by flow cytometry. Data presented are from three separate experiments.
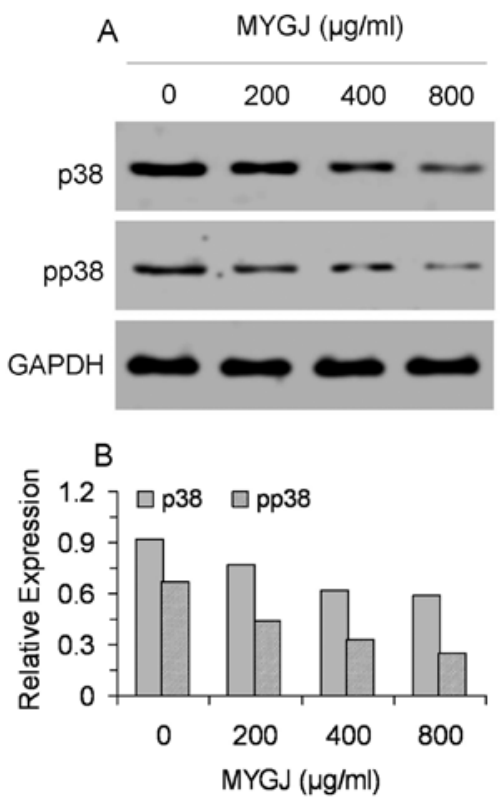

Figure 5. MYGJ inhibited p38 MAPK expression in Bel-7402 cells. (A) Suspension-cultured Bel-7402 cells were collected after MYGJ treatment, and subjected to Western blotting using antibody against p38 MAPK (p38), phospho-p38 MAPK (pp38) or GAPDH. (B) Densitometric analysis of p38 protein level after normalization with GAPDH protein level.

by the caspase (cysteine aspartate-specific proteinase) cascade $(21,22)$. To determine whether caspases contributed to MYGJinduced apoptosis in Bel-7402 cells during suspension growth, activities of caspase-3, -8 and -9 were detected. As shown in Fig. 4A-C, MYGJ activated caspase-3, -8 and -9 in a dosedependent manner, and compared with controls $(\mathrm{P}<0.01)$. In addition, the Z-VAD-FMK pan caspase inhibitor, significantly inhibited anoikis in Bel-7402 cells elicited by MYGJ, which indicated that the MYGJ's effect was correlated with caspase activation.
MYGJ inhibited p38 MAPK expression in Bel-7402 cells. It has been reported that MAPK signal pathway was involved in 'detachment-induced apoptosis-anoikis' $(23,24)$. We further observed the effects of Bel-7402 cells on p38 MAPK expression. As shown in Fig. 5, MYGJ inhibited the expression and phosphorylation of p38 MAPK in Bel-7402 cells, suggesting the effects of MYGJ might be correlated with p38 MAPK.

\section{Discussion}

Epithelial cells require attachment to the extracellular matrix to provide a survival signal. Detachment from the extracellular matrix causes apoptosis, a process known as anoikis. Anoikis is a Greek word that means homelessness, as apoptotic process, anoikis was first discovered by Frisch in 1994 (25). Physiologically, anoikis played a critical role in organismal development and homeostasis (25). Pathophysiologically, resistance to anoikis is acquired in cancer cells due to gene expression or activity abnormality, which allows partial cancer cells to survive when deprived of extracellular matrix attachment during dissemination in blood or lymphs, and associated with metastatic spread of cancer cells. Anoikis has been suggested as a potential target for anti-cancer therapy $(22,23)$. Poly-HEMA, a non-toxic polymer of 2-hydroxyethyl methacrylate, was extensively utilized to mimic anoikis in vitro because of its ability to reduce tissue culture plastic adhesivity $(22,26,27)$.

Our present results revealed that MYGJ can inhibit Bel-7402 cells proliferation in the poly-HEMA mimicked detachment from the extracellular matrix. Meanwhile, the concentration of MYGJ used in Bel-7402 cells showed no significant effects against proliferation in normal hepatocyte HL-7702 cells, and may excluded the non-specific cytotoxicity of MYGJ in vitro. Soft agar colony formation assay further demonstrated that MYGJ significantly inhibited colony forming activity of Bel-7402 cells in soft agar, which suggested MYGJ could inhibit anchorage-independent growth 
of Bel-7402 cells. Furthermore, anoikis was detected by use of EthD-1 (Ethidium homodimer) as indicator. EthD-1 is a highaffinity nucleic acid fluorescent dye, which can only penetrate cells once the membrane is damaged (a hallmark of dead cells) and produces bright red fluorescence upon binding to nucleic acids, and could be used to detect anoikis. In the present study, EthD-1 staining indicated that partial Bel-7402 cells absorbed EthD-1 and emitted red fluorescence after MYGJ treatment. In addition, nuclear fragmentation was also observed in Bel-7402 cells. MYGJ also activated caspase-3, which indicated that MYGJ could promote the anoikis of Bel-7402 cells. These observations suggest that MYGJ may induce anoikis in Bel-7402 cells.

There is a growing consensus that lack of signalling through the MAPK/ERK pathway, through loss of integrinmediated contact with the extracellular matrix, contributes to anoikis $(22,24,28-30)$. Integrins, the main cell surface receptors 'sense' adhesion to the extracellular matrix, triggering an intracellular signaling cascade via focal adhesion kinase (FAK) and other kinases, leading to activation of the MAP kinases. The signal transduction from extracellular matrix to MAPK signal is inhibited after the separation of adherence to extracellular matrix, which results in impaired regulation of apoptosis and eventually cell death. Similar to classic apoptosis, anoikis is also executed by intracellular caspases that are activated during the onset of apoptosis by extrinsic and intrinsic pathways $(21,22)$. The extrinsic pathway involves oligomerization of cell-surface death receptors by their ligands, resulting in recruitment and activation of caspase- 8 followed by activation of executioner caspases-3. The intrinsic pathway involves the signals to mitochondria, which lead to the release of cytochrome c, Apaf-1, forming an apoptosome that activates the initiating protease caspase- 9 which in turn activates executioner caspase-3, causing the cell to undergo apoptosis. Our findings demonstrated that MYGJ inhibited expression and phosphorylation of p38 MAPK, and activated caspase-3, -8 and -9 in Bel-7402 cells. Blocking caspases activity completely inhibited the anoikis induced by MYGJ. The present observations suggest that the anoikis of Bel-7402 cells, induced by MYGJ, was related to p38 MAPK, and both the intrinsic and extrinsic apoptotic pathway.

In conclusion, our findings demonstrated that MYGJ could inhibit growth suspension and induce anoikis in human hepatocarcinoma Bel-7402 cells in vitro. The effects of MYGJ might be related to the inhibition expression and phosphorylation of p38 MAPK, and intrinsic and extrinsic pathways of apoptosis. Additional studies are needed to determine whether MYGJ plays a role in hepatocarcinoma cells in blood or lymphatic circulation. The present study also provides insight into the application of the ancient Chinese Herbal formulas.

\section{Acknowledgements}

This study was partially supported by Key Basic Research Program from Science and Technology Commission of Shanghai Municipality (09JC1413600), Key Discipline of State Administration of Traditional Chinese Medicine (Traditional Chinese Medicine in Oncology), E-institutes of Shanghai Municipal Education Commission (E 03008), and Shanghai Shen Kang Platform Grant (SHDC12007206). We also thank
Shanghai Key Laboratory of Tissue Engineering for technical assistance.

\section{References}

1. Jemal A, Bray F, Center MM, Ferlay J, Ward E and Forman D: Global cancer statistics. CA Cancer J Clin 61: 69-90, 2011.

2. Hagymási $\mathrm{K}$ and Tulassay Z: Epidemiology risk factors and molecular pathogenesis of primary liver cancer. Orv Hetil 149: 541-548, 2008.

3. Rampone B, Schiavone B and Confuorto G: Current management of hepatocellular cancer. Curr Oncol Rep 12: 186-192, 2010.

4. Lord R, Suddle A and Ross PJ: Emerging strategies in the treatment of advanced hepatocellular carcinoma: the role of targeted therapies. Int J Clin Pract 65: 182-188, 2011.

5. Wysocki PJ: Targeted therapy of hepatocellular cancer. Expert Opin Investig Drugs 19: 265-274, 2010.

6. Yu Y, Lang Q, Chen Z, Li B, Yu C, Zhu D, Zhai X and Ling C: The efficacy for unresectable hepatocellular carcinoma may be improved by transcatheter arterial chemoembolization in combination with a traditional Chinese herbal medicine formula: a retrospective study. Cancer 115: 5132-5138, 2009.

7. Meng MB, Cui YL, Guan YS, Ying Z, Zheng MH, Yuan CK and Zhang RM: Traditional Chinese medicine plus transcatheter arterial chemoembolization for unresectable hepatocellular carcinoma. J Altern Complement Med 14: 1027-1042, 2008.

8. Shu X, McCulloch M, Xiao H, Broffman M and Gao J: Chinese herbal medicine and chemotherapy in the treatment of hepatocellular carcinoma: a meta-analysis of randomized controlled trials. Integr Cancer Ther 4: 219-229, 2005.

9. Du Q, Hu B, Shen KP and An HM: Progress in TCM pathogenesis and treatment of liver cancer. World J Integr Tradit West Med 5: 814-817, 2010 (In Chinese).

10. Hou FG, Ling CQ, Zhao G and He XM: Investigation of clinical distribution of basic TCM Syndrome in primary hepatic carcinoma. Shanghai J Tradit Chinese Med 39: 22-23, 2005 (In Chinese).

11. Wu YF, Wang SP, Sun JM and Wei JN: Clinical distribution and standards of TCM Syndrome of primary hepatic carcinoma. J Shanxi Coll Tradit Chinese Med 8: 21-23, 2007 (In Chinese).

12. Pan MQ, Zeng PH and Pan B: Regularity of traditional Chinese medicine in middle and advanced primary hepatic carcinoma. Chinese Arch Tradit Chinese Med 21: 1641-1642, 2003 (In Chinese).

13. Liu Q, Zhang YB, Ma CH, Yue XQ and Ling CQ: Analysis of literature on therapeutic methods and medicines of traditional Chinese medicine for primary liver cancer. Zhong Xi Yi Jie He Xue Bao 3: 260-262, 2005 (In Chinese).

14. Zhang M, Chen H, Huang J, Li Z, Zhu C and Zhang S: Effect of lycium barbarum polysaccharide on human hepatoma QGY7703 cells: inhibition of proliferation and induction of apoptosis. Life Sci 76: 2115-2124, 2005.

15. Tsai NM, Lin SZ, Lee CC, Chen SP, Su HC, Chang WL and Harn HJ: The antitumor effects of angelica sinensis on malignant brain tumors in vitro and in vivo. Clin Cancer Res 11: 3475-3484, 2005.

16. Tang MZ, Wang ZF and Shi YL: Involvement of cytochrome c release and caspase activation in toosendanin-induced PC12 cell apoptosis. Toxicology 201: 31-38, 2004.

17. Du Q, Hu B and Shen KP: A Survey of anti-cancer effects of Chinese tonifying herbs in primary hepatic carcinoma. J Chin Med Mater 33: 1512-1516, 2010 (In Chinese).

18. Hsu YL, Kuo PL, Tzeng TF, Sung SC, Yen MH, Lin LT and Lin CC: Huang-lian-jie-du-tang, a traditional Chinese medicine prescription, induces cell-cycle arrest and apoptosis in human liver cancer cells in vitro and in vivo. J Gastroenterol Hepatol 23: E290-E299, 2008.

19. Hu B, Shen KP, An HM, Wu Y and Du Q: Aqueous extract of Curcuma aromatica induces apoptosis and G2/M arrest in human colon carcinoma LS-174-T cells independent of p53. Cancer Biother Radiopharm 26: 97-104, 2011.

20. Hu B, An HM, Shen KP and DU Q: Effects of Tenglong Buzhong Decoction on proliferation and apoptosis of human colon carcinoma cell line LS174T. Zhong Xi Yi Jie He Xue Bao 8: 575-580, 2010 (In Chinese).

21. Chiarugi P and Giannoni E: Anoikis: a necessary death program for anchorage-dependent cells. Biochem Pharmacol 76: 1352-1364, 2008. 
22. Simpson CD, Anyiwe $K$ and Schimmer AD: Anoikis resistance and tumor metastasis. Cancer Lett 272: 177-185, 2008.

23. Geiger TR and Peeper DS: Metastasis mechanisms. Biochim Biophys Acta 1796: 293-308, 2009.

24. Owens TW, Valentijn AJ, Upton JP, Keeble J, Zhang L, Lindsay J, Zouq NK and Gilmore AP: Apoptosis commitment and activation of mitochondrial Bax during anoikis is regulated by p38MAPK. Cell Death Differ 16: 1551-1562, 2009.

25. Frisch SM and Francis H: Disruption of epithelial cell matrix interactions induces apoptosis. J Cell Biol 124: 619-626, 1994.

26. Sakamoto S, McCann RO, Dhir R and Kyprianou N: Talin1 promotes tumor invasion and metastasis via focal adhesion signaling and anoikis resistance. Cancer Res 70: 1885-1895, 2010 .
27. Kochetkova M, Kumar S and McColl SR: Chemokine receptors CXCR4 and CCR7 promote metastasis by preventing anoikis in cancer cells. Cell Death Differ 16: 664-673, 2009.

28. Uehara N, Matsuoka Y and Tsubura A: Mesothelin promotes anchorage-independent growth and prevents anoikis via extracellular signal-regulated kinase signaling pathway in human breast cancer cells. Mol Cancer Res 6: 186-193, 2008.

29. Guo W and Giancotti FG: Integrin signalling during tumour progression. Nat Rev Mol Cell Biol 5: 816-826, 2004.

30. Nagaprashantha LD, Vatsyayan R, Lelsani PC, Awasthi S and Singhal SS: The sensors and regulators of cell-matrix surveillance in anoikis resistance of tumors. Int J Cancer 128: 743-752, 2001. 